\title{
SOME LIMIT THEOREMS
}

I. M. SHEFFER

1. Introduction. It is a classical result in the theory of trigonometric series that if

$$
c_{n} \cos n x+d_{n} \sin n x \rightarrow 0
$$$$
(n \rightarrow \infty)
$$

for all (real) $x$ on a set of positive measure, then

$$
c_{n} \rightarrow 0, \quad d_{n} \rightarrow 0 .
$$

Cantor proved this for the case that set $\{x\}$ is an interval, and Lebesgue established the result for a set of measure zero. A short proof is given by Hardy and Rogosinski. ${ }^{1}$

The following related result was proved and used by Szász. ${ }^{2}$ If

$$
a_{n} \sin n x+b_{n} \sin (n+1) x \rightarrow 0
$$

on a (real) set $\{x\}$ of positive measure, then

$$
a_{n} \rightarrow 0, \quad b_{n} \rightarrow 0 .
$$

Relations (1.1) and (1.3) can be put into complex form. For example, (1.1) becomes

$$
a_{n} \exp \{n x\}+b_{n} \exp \{-n x\} \rightarrow 0,
$$

with the conclusion that

$$
a_{n} \rightarrow 0, \quad b_{n} \rightarrow 0 .
$$

Here $\exp \{u\}$ is defined by

$$
\exp \{u\} \equiv e^{i u} \quad\left(i=(-1)^{1 / 2}\right) .
$$

Our purpose in the present work is to extend the conclusions of the above-mentioned results to combinations more general than (1.3), (1.5). Thus in $\S 2$ we go from two terms to $k$ terms and generalize the exponents; in $\S 3$ the coefficients of the exponentials are permitted

Presented to the Society, April 26, 1947 under the title $A$ limit theorem; received by the editors May 12, 1947.

${ }^{1}$ Hardy and Rogosinski, Fourier series (Cambridge Tracts in Mathematics and Mathematical Physics, no. 38), Theorem 92, p. 84.

2 Otto Szász, On Lebesgue summability and its generalization to integrals, Amer. J. Math. vol. 67 (1945) pp. 389-396, especially Lemma 2, p. 395. Dr. Szász has informed me that, with the intention of using it in work on trigonometric series, he has proved (but not published) a generalization of (1.3), namely where the left side of (1.3) is replaced by the expression $\sum_{s-n}^{n+k} a_{s} e^{i s x}$. 
to be polynomials; and in $\S 4$ the multi-dimensional case is taken up.

2. One-dimensional case. In the following sections we suppose without further mention that all variables $x, y, \ldots$ are real.

LEMMA 2.1. Let $\left\{u_{n}\right\}$ be a real sequence that does not have zero as its limit. The relation

$$
\lim _{n \rightarrow \infty} \exp \left\{u_{n} x\right\}=1
$$

cannot hold on a set of positive measure. ${ }^{3}$

Suppose the lemma is false, so that there is a set $\mathcal{F}$ of positive measure on which (2.1) is satisfied. We may suppose that $\mathcal{F}$ is bounded. It is no restriction to assume that zero is not a limit point of $\left\{u_{n}\right\}$; for there exists an infinite subsequence of $\left\{u_{n}\right\}$ for which zero is not a limit point, and we may remove all $u_{n}$ 's not in this subsequence.

Suppose $\left\{u_{n}\right\}$ contains a bounded subsequence $\left\{u_{n_{j}}\right\}$; then from $\left\{u_{n_{j}}\right\}$ a further sequence can be chosen for which a limit exists. This limit, say $L$, cannot be zero, so from $\exp \{L x\}=1$ for $x$ in $\mathcal{F}$ we conclude that $\mathcal{F}$ is at most a denumerable set, contrary to the assumption that $\mathcal{F}$ is of positive measure.

Now suppose that $\left\{u_{n}\right\}$ contains no bounded subsequence, so that $\left|u_{n}\right| \rightarrow \infty$. By Egoroff's theorem there is a subset $\mathcal{F}_{1}$ of $\mathcal{F}$, of positive measure, on which (2.1), that is,

$$
\cos u_{n} x+i \sin u_{n} x \rightarrow 1 \text {, }
$$

holds uniformly. Consequently, since $1+\cos u_{n} x$ is uniformly bounded,

$$
\cos ^{2} u_{n} x-1 \rightarrow 0 \quad \text { (uniformly on } \mathcal{f}_{1} \text { ). }
$$

Integrating over $\mathfrak{F}_{1}$ :

$$
\int_{\mathcal{F}_{1}} \cos ^{2} u_{n} x d x \rightarrow \int_{\mathcal{F}_{1}} d x=m\left(\mathcal{F}_{1}\right) .
$$

Now there exists an open set 2, consisting of a finite number of nonoverlapping intervals, say $\left(a_{j}, b_{j}\right), j=1, \cdots, p$, with the following two properties: (i) 2 contains $\mathcal{f}_{1}$; (ii) $m\left(\mathcal{f}_{1}\right) \leqq m(2)<3 m\left(\mathcal{f}_{1}\right) / 2$. Hence,

${ }^{3}$ As originally stated and proved, this and the other results of the paper used the condition interval everywhere in place of set of positive measure. We owe to Dr. Szász the suggestion to generalize to the case of positive measure, and also to extend the results to more than one variable (see $\$ 4$ ). 


$$
\begin{aligned}
& \int_{\mathcal{F}_{1}} \cos ^{2} u_{n} x d x \leqq \int_{Q^{2}} \cos ^{2} u_{n} x d x=2^{-1} \int_{Q^{2}}\left(1+\cos 2 u_{n} x\right) d x \\
& =2^{-1} m(2)+2^{-1} \sum_{j=1}^{p}\left[\frac{\sin 2 u_{n} x}{2 u_{n}}\right]_{a j}^{b_{j}} ;
\end{aligned}
$$

so for all $n$ sufficiently large,

$$
\int_{\mathcal{F}_{1}} \cos ^{2} u_{n} x d x<\frac{3}{4} m\left(\mathscr{F}_{1}\right) .
$$

But this contradicts (2.2), so the lemma cannot be false. ${ }^{4}$

LeMmA 2.2. Let $\left\{t_{s, n}\right\}, s=1, \cdots, k$, be real sequences with the property that none of the sequences $\left\{t_{s, n}\right\},\left\{t_{s, n}-t_{p, n}\right\}(s \neq p)$ has zero as a limit point. If real or complex constants $\left\{A_{s, n}\right\}$ exist such that

$$
\sum_{s=1}^{k} A_{s, n}\left[\exp \left\{t_{s, n} x\right\}-1\right] \rightarrow 0
$$

for all $x$ on a set $\varepsilon$ of positive measure, then

$$
A_{s, n} \rightarrow 0 \quad(s=1, \cdots, k) .
$$

If $k=1$ the lemma is true in virtue of Lemma 2.1. Assume it true for the case $k-1$; we shall then prove it for $k$ by an induction argument, and this will establish the truth of Lemma 2.2.

It is no restriction to suppose that $\varepsilon$ is a bounded set. $\varepsilon$ contains a point $x_{1}$ with the property that every interval containing $x_{1}$ in its interior meets $\varepsilon$ in a set of positive measure. ${ }^{5}$ For suppose not. Then about each $x$ in $\varepsilon$ exists an interval $I_{x}$, with $x$ in its interior, such that $\mathcal{E} \cdot I_{x}$ is of measure zero. Let $x_{1}$ be in $\varepsilon$, and let $I_{x_{1}}$ be the largest associated interval. It is clear that there is a largest interval. If $x_{2}$ in $\varepsilon$ is not in $I_{x_{1}}$, then it too has a largest associated interval $I_{x_{2}}$, and $I_{x_{1}}$, $I_{x_{2}}$ do not meet. It is now a straightforward argument to show that $\varepsilon$ is covered by at most a denumerable number of such intervals $I_{x}$, thus establishing $\varepsilon$ as a set of zero measure. This contradiction shows that a point such as the aforementioned $x_{1}$ exists.

Let $x$ in (2.4) take on such a value $x_{1}$ and subtract from (2.4). There results the relation

1 This contradiction does not preclude the possibility that the set of points for which (2.1) holds is non-measurable, but in this case the set cannot contain a subset of positive measure.

5 A much stronger conclusion as to the number of such points $x_{1}$ is of course possible, but we require only the above mild assertion. 


$$
\sum_{s=1}^{k} A_{s, n} \exp \left\{t_{s, n} x_{1}\right\}\left[\exp \left\{t_{s, n} y\right\}-1\right] \rightarrow 0
$$

for all $y$ in $\varepsilon_{1} \equiv\left\{y=x-x_{1}\right.$, as $x$ ranges over $\left.\varepsilon\right\} . \varepsilon_{1}$ and $\varepsilon$ have the same (positive) measure, and the replacement of (2.4) and $\varepsilon$ by (2.6) and $\varepsilon_{1}$ insures that the origin $(y=0)$ is a point of $\varepsilon_{1}$ every neighborhood of which contains a subset of $\varepsilon_{1}$ of positive measure.

Let

$$
B_{s, n}=A_{s, n} \exp \left\{t_{s, n} x_{1}\right\}
$$

Then

$$
\sum_{s=1}^{k} B_{s, n}\left[\exp \left\{t_{s, n} y\right\}-1\right] \rightarrow 0 \quad\left(y \text { in } \varepsilon_{1}\right),
$$

and (2.5) is equivalent to

$$
B_{s, n} \rightarrow 0 \quad(s=1, \cdots, k) .
$$

Suppose the lemma is false for case $k$. Then a value $s$, say $s=k$, exists such that $t^{6} B \rightarrow 0$ is false. There therefore is a subsequence $\left\{n_{j} \equiv n(j)\right\}$ of $\{n\}$, and a number $M>0$, such that

$$
\left|B_{k, n(j)}\right|>M
$$

so on replacing $\{n\}$ by $\{n(j)\}$ in (2.8) and dividing by $B_{k, n(j)}$, we have

$$
\left[\exp \left\{t_{k, n(j)} y\right\}-1\right]+\sum_{s=1}^{k-1} C_{s, n(j)}\left[\exp \left\{t_{s, n(j)} y\right\}-1\right] \rightarrow 0
$$

Here

$\left(y\right.$ in $\left.\varepsilon_{1}\right)$.

$$
C_{s, n(j)}=\frac{B_{s, n(j)}}{B_{k, n(j)}}
$$

Let $y_{1}$ be an arbitrary point of $\varepsilon_{1}$. The set of points $\{u\}$ defined by $u=y-y_{1}$ as $y$ ranges over $\varepsilon_{1}$ will be denoted by $\varepsilon_{y_{1}}$, and will be termed a translation set (relative to $\varepsilon_{1}$ ). Clearly, $m\left(\varepsilon_{y_{1}}\right)=m\left(\varepsilon_{1}\right)$.

Let 2 be an open set containing $\varepsilon_{1}$, with $m\left(\varepsilon_{1}\right)<m(2)<3 m\left(\varepsilon_{1}\right) / 2$. There exists a set of nonoverlapping open intervals $2_{1}=I_{1}+\cdots+I_{r}$ contained in 2, for which

${ }^{6}$ Actually, for every $s=1, \cdots, k$ the quantity $B_{s, n}$ does not approach zero; for otherwise we can drop from sum (2.8) all terms for which $B_{8, n} \rightarrow 0$, and thus reduce (2.8) to $k-1$ or less terms, in which case the lemma is true by our induction assumption, 


$$
m\left(\varepsilon_{1}\right)<m\left(2_{1}\right)<3 m\left(\varepsilon_{1}\right) / 2
$$

and also such that

$$
m\left(Q_{1} \cdot \varepsilon_{1}\right)>3 m\left(\varepsilon_{1}\right) / 4 \text {. }
$$

To each end of $I_{p}(p=1, \cdots, r)$ add an interval of length $\Delta$, forming a new interval $J_{p}$, where $\Delta$ is chosen small enough so that on setting $2_{2}=J_{1}+\cdots+J_{q}(q \leqq r$ since some intervals may overlap and thus be combined) then $m\left(Q_{2}\right)<3 m\left(\varepsilon_{1}\right) / 2$.

Let $\mathcal{H}$ be the subset of numbers $y$ of $\varepsilon_{1}$ for which $|y|<\Delta$. We know that $\mathfrak{H C}$ is of positive measure. Moreover, for an arbitrary $y$ in $\mathfrak{H}$,

$$
m\left(\mathscr{Q}_{2} \cdot \varepsilon_{y}\right)>3 m\left(\varepsilon_{1}\right) / 4, \quad m\left(2_{2} \cdot \varepsilon_{1}\right)>3 m\left(\varepsilon_{1}\right) / 4
$$

Since $m\left(2_{2}\right)<3 m\left(\varepsilon_{1}\right) / 2$, it follows that

$$
m\left(\varepsilon_{1} \cdot \varepsilon_{y}\right)>0
$$

(all $y$ in $\mathfrak{H C}$ ).

We see from (2.10) and Lemma 2.1 that we cannot have $C_{s, n(j)} \rightarrow 0$ for all $s=1, \cdots, k-1$. Hence there is an $s$, say $s=1$, for which $C_{1, n(j)} \rightarrow 0$ is false; and a subsequence $\{m(j)\}$ of $\{n(j)\}$, and a positive number $K$, such that

$$
\left|C_{1, m(j)}\right|>K
$$

Now the relation

$$
\exp \left\{\left(t_{1, m(j)}-t_{k, m(j)}\right) z\right\} \rightarrow 1
$$

cannot hold on a set of positive measure (Lemma 2.1); consequently, there is a point $y_{1}$ in $\mathfrak{H}$ such that (2.13) is false for $z=y_{1}$. Choose $y=y_{1}$ in (2.10) and subtract from (2.10):

$$
\begin{aligned}
\exp \left\{t_{k, n(j)} y_{1}\right\} & {\left[\exp \left\{t_{k, n(j)} u\right\}-1\right] } \\
+ & \sum_{s=1}^{k-1} C_{s, n(j)} \exp \left\{t_{s, n(j)} y_{1}\right\}\left[\exp \left\{t_{s, n(j)} u\right\}-1\right] \rightarrow 0,
\end{aligned}
$$

where $u=y-y_{1}\left(y\right.$ in $\varepsilon_{1}$ ), so that $u$ ranges over the translation set $\varepsilon_{y_{1}}$. In (2.14), replace $\{n(j)\}$ by $\{m(j)\}$ and divide by $\exp \left\{t_{k, m(j)} y_{1}\right\}$. This gives us

$$
\begin{array}{r}
{\left[\exp \left\{t_{k, m(j)} u\right\}-1\right]} \\
(2.15)+\sum_{s=1}^{k-1} C_{s, m(j)} \exp \left\{\left(t_{s, m(j)}-t_{k, m(j)}\right) y_{1}\right\}\left[\exp \left\{t_{s, m(j)} u\right\}-1\right] \rightarrow 0 \\
\quad\left(u \text { in } \varepsilon_{y_{1}}\right) .
\end{array}
$$

Let $\mathcal{L}=\varepsilon_{1} \cdot \varepsilon_{y_{1}}$. We know that $m(\mathcal{L})>0$. If we restrict $u$ in $(2.15)$ 
and $y$ in (2.10) to lie in $\mathcal{L}$, then $u$ and $y$ may be identified; so on subtracting (2.15) from $(2.10)$ (with $n(j)$ replaced by $m(j)$ ) we obtain

$$
\sum_{s=1}^{k-1} C_{s, m(j)}\left[1-\exp \left\{\left(t_{s, m(j)}-t_{k, m(j)}\right) y_{1}\right\}\right]\left[\exp \left\{t_{s, m(j)} y\right\}-1\right] \rightarrow 0
$$

$(y$ in $\mathcal{L})$.

This relation has only $k-1$ terms, and for it the hypotheses of Lemma 2.2 hold. By our induction assumption, therefore, each coefficient approaches zero. Since (2.12) holds, we must have

$$
\exp \left\{\left(t_{1, m(j)}-t_{k, m(j)}\right) y_{1}\right\} \rightarrow 1,
$$

which is contrary to the choice of $y_{1}$.

Thus the induction chain is complete, and the lemma is established.

TheOREM 2.1. Let $\left\{a_{s, n}\right\}, s=1, \cdots, k$, be real or complex number sequences, and let the real sequences $\left\{r_{s, n}\right\}$ have the property that none of the sequences $\left\{r_{s, n}-r_{p, n}\right\}(s \neq p)$ has zero as a limit point. If

$$
\sum_{s=1}^{k} a_{s, n} \exp \left\{r_{s, n} x\right\} \rightarrow 0
$$

for all $x$ on a set $\mathcal{E}$ of positive measure, then

$$
a_{s, n} \rightarrow 0 \quad(s=1, \cdots, k) .
$$

REMARK. For sequences $\left\{r_{s, n}\right\}$ satisfying the above hypothesis, Theorem 2.1 asserts what may be termed the asymptotic linear independence of the functions $\exp \left\{r_{s, n} x\right\}, s=1, \cdots, k$.

If the theorem is false, there is an index $s$, say $s=1$, for which $a_{1, n} \rightarrow 0$ is false; so a subsequence $\{n(j)\}$ of $\{n\}$ exists, and a positive number $M$, such that $\left|a_{1, n(j)}\right|>M$. Replace $\{n\}$ by $\{n(j)\}$ in (2.17) and divide by $a_{1, n(j)} \exp \left\{r_{1, n(j)} x\right\}$ :

$$
1+\sum_{s=2}^{k} b_{s, n(j)} \exp \left\{\left(r_{s, n(j)}-r_{1, n(j)}\right) x\right\} \rightarrow 0,
$$

where

$$
b_{s, n(j)}=\frac{a_{s, n(j)}}{a_{1, n(j)}} .
$$

Take $x=x_{1}$ in (2.19) and subtract from (2.19):

$$
\sum_{s=2}^{k} b_{s, n(j)} \exp \left\{t_{s, n(j)} x_{1}\right\}\left[\exp \left\{t_{s, n(j)} y\right\}-1\right] \rightarrow 0 .
$$


Here

$$
t_{s, n(j)}=r_{s, n(j)}-r_{1, n(j)} ; y=x-x_{1} \quad(x \text { in } \varepsilon),
$$

so $y$ ranges over a set $\varepsilon_{1}$ of positive measure.

The hypotheses of Lemma 2.2 are satisfied, so

$$
b_{s, n(j)} \rightarrow 0 \quad(s=2, \cdots, k) .
$$

But this contradicts (2.19). Thus Theorem 2.1 is established.

COROLLARY 2.1. Let the sequences $\left\{t_{s, n}\right\}$ satisfy the hypothesis of Lemma 2.2. If a constant $A$ and constants $\left\{a_{s, n}\right\}$ exist such that

$$
\sum_{s=1}^{k} a_{8, n} \exp \left\{t_{8, n} x\right\} \rightarrow A
$$

for all $x$ on $a$ set of positive measure, then

$$
A=0 ; \quad a_{s, n} \rightarrow 0 \quad(s=1, \cdots, k) .
$$

For, (2.22) can be written

$$
\sum_{s=1}^{k+1} a_{s, n} \exp \left\{t_{s, n} x\right\} \rightarrow 0,
$$

where

$$
a_{k+1, n} \equiv-A, \quad t_{k+1, n} \equiv 0 .
$$

The hypothesis of Theorem 2.1 is fulfilled in (2.24), so $a_{s, n} \rightarrow 0$, $s=1, \cdots, k+1$.

In Theorem 2.1 the condition on the sequences $\left\{r_{s, n}\right\}$ cannot be weakened. This is shown by the following theorem.

THEOREM 2.2. Let the real sequences $\left\{r_{s, n}\right\}, s=1, \cdots, k$, be such that at least one of the sequences $\left\{r_{s, n}-r_{p, n}\right\}(s \neq p)$ has zero as a limit point. There exist sequences $\left\{a_{s, n}\right\}$, at least one of which does not approach zero, such that (2.17) holds for all $x$.

We may suppose that $t_{n(j)} \equiv r_{1, n(j)}-r_{2, n(j)} \rightarrow 0$ as $j \rightarrow \infty$. Choose $a_{s, n}=0, s=3, \cdots, k$, and $a_{1, n}=a_{2, n}=0$ for $n \neq n_{1}, n_{2}, \cdots$ The left side of (2.17) becomes

$$
a_{1, n(j)} \exp \left\{r_{1, n(j)} x\right\}+a_{2, n(j)} \exp \left\{r_{2, n(j)} x\right\},
$$

and this approaches zero if and only if

$$
a_{1, n(j)} \exp \left\{t_{n(j)} x\right\}+a_{2, n(j)} \rightarrow 0 .
$$

It is clear that if we define $a_{1, n(j)}=1, a_{2, n(j)}=-1$, then (2.27) 
does hold for every $x$. Actually, these coefficients can be chosen to be unbounded. For let $R>0$ be given. There exists an $M=M(R)$ such that

$$
\left|\exp \left\{t_{n(j)} x\right\}-1\right| \leqq M\left|t_{n(j)}\right|
$$

for all $|x| \leqq R$. On writing the left side of (2.27) as

$$
a_{1, n(j)}\left[\exp \left\{t_{n(j)} x\right\}-1\right]+\left[a_{1, n(j)}+a_{2, n(j)}\right],
$$

we see that (2.27) follows if we choose $a_{1, n(j)}, a_{2, n(j)}$ so that

$$
a_{1, n(j)} t_{n(j)} \rightarrow 0, \quad a_{1, n(j)}+a_{2, n(j)} \rightarrow 0 .
$$

Since $t_{n(j)} \rightarrow 0$, conditions (2.28) can be satisfied by sequences $a_{1, n(j)}$, $a_{2, n(j)}$ that are unbounded.

3. Polynomial coefficients. The result of Theorem 2.1 can be extended to the case of polynomial coefficients of bounded degree:

THEOREM 3.1. Let $\left\{r_{s, n}\right\}, s=1, \cdots, k$, be real sequences such that none of the sequences $\left\{r_{s, n}-r_{p, n}\right\}(s \neq p)$ has zero as a limit point. Let $\left\{P_{s, n}(x)\right\}$ be real or complex polynomial sequences:

$$
P_{s, n}(x)=a_{s, 0, n}+a_{s, 1, n} x+\cdots+a_{s, q_{s}, n} x^{q_{s}} \quad(s=1, \cdots, k)
$$

in which $q_{s}$ is independent of $n$. If

$$
\sum_{s=1}^{k} P_{s, n}(x) \exp \left\{r_{s, n} x\right\} \rightarrow 0
$$

for all $x$ on a set $\mathcal{E}$ of positive measure, then

$$
a_{s, p, n} \rightarrow 0 \quad\left(p=0,1, \cdots, q_{s} ; s=1, \cdots, k\right) .
$$

Let

$$
q=\max \left\{q_{1}, \cdots, q_{k}\right\} .
$$

If $q=0$ the result follows from Theorem 2.1. Suppose the theorem is false. Then there is an integer $Q>0$ such that whenever $q<Q$ the result is true, but for at least one case with $q=Q$ the theorem is untrue. In each case of failure, with $q=Q$, at least one polynomial coefficient is of degree $Q$. Let $\lambda$ be the number of such polynomials; then there is a positive integer $\Lambda$ with the property that whenever $\lambda<\Lambda$ (and $q=Q$ ), the theorem is true, but there is a case $\lambda=\Lambda$, $q=Q$ for which it is false.

Let (3.2) be such a case, so that exactly $\Lambda$ polynomials, that we may take to be $P_{s, n}(x), s=1, \cdots, \Lambda$, are of degree $Q$ while all other polynomial coefficients (if any) are of lower degree. Since the theorem is 
false for this case, not all the coefficients approach zero as $n \rightarrow \infty$. For each $n=1,2, \cdots$ let

$$
\mu_{n}=\max \left\{\left|a_{s, p, n}\right|\right\} \quad\left(p=0,1, \cdots, q_{s} ; s=1, \cdots, k\right) .
$$

Then $\mu_{n}$ does not approach 0 . There therefore exist values $s=\sigma, p=p_{\sigma}$, a positive number $M$, and a subsequence $\{n(j)\}$ of $\{n\}$, such that

$$
\left|a_{\sigma, p_{\sigma}, n(j)}\right|=\mu_{n(j)}>M \quad(j=1,2, \cdots) .
$$

Replace $\{n\}$ by $\{n(j)\}$ in (3.2) and divide by $a_{\sigma, p_{\sigma}, n(j)} \exp \left\{r_{1, n(j)} x\right\}$ :

$$
R_{1, n(j)}(x)+\sum_{s=2}^{k} R_{s, n(j)}(x) \exp \left\{t_{s, n(j)} x\right\} \rightarrow 0 \quad(x \text { in } \varepsilon),
$$

where

$$
t_{s, n(j)}=r_{s, n(j)}-r_{1, n(j)}
$$

and

$$
R_{s, n(j)}(x)=\sum_{p=0}^{q_{s}} b_{s, p, n(j)} x^{p} \equiv \frac{1}{a_{\sigma, p_{\sigma}, n(j)}} \cdot P_{s, n(j)}(x) .
$$

The $b$-coefficients are bounded, and $b_{\sigma, r_{\sigma}, n(j)} \equiv 1$ for all $j$. Consequently, there exists a subsequence $\{m(j)\}$ of $\{n(j)\}$ for which the following limits exist:

$$
\lim _{j \rightarrow \infty} b_{s, p, m(j)}=b_{s, p} \quad\left(p=0,1, \cdots, q_{s} ; s=1, \cdots, k\right) ;
$$

and not all of $b_{s, p}$ are zero, since $b_{\sigma, p_{\sigma}}=1$.

From (3.7) it follows that

$$
R_{1}(x)+\sum_{s=2}^{k} R_{s}(x) \exp \left\{t_{s m(j)} x\right\} \rightarrow 0 \quad(x \text { in } \varepsilon),
$$

where

$$
R_{s}(x)=\sum_{p=0}^{q_{s}} b_{s, p} x^{p} \quad(s=1, \cdots, k) .
$$

Moreover, $R_{1}(x)$ is of degree $Q$; for if it is of lower degree, then (3.9) presents a case in which fewer than $\Lambda$ polynomials are of degeee $Q$, so from the definition of $\Lambda$ it will follow that the theorem is true for (3.9). Thus all coefficients in all the polynomials approach zero as $j \rightarrow \infty$. But this is contrary to the fact that $b_{\sigma, p_{\sigma}}=1$. Hence the degree of $R_{1}(x)$ must be $Q$.

We know from the proof of Lemma 2.2 that set $\varepsilon$ contains a point 
$x_{1}$ every neighborhood of which contains a subset of $\varepsilon$ of positive measure; and using this fact, we may conclude (as was similarly argued in establishing Lemma 2.2 ) that there exist distinct numbers $h_{1}, h_{2}$ such that on defining $\varepsilon_{1}, \varepsilon_{2}$ by

$$
\varepsilon_{p} \equiv\left\{y_{p}=x+h_{p}, x \text { ranging over } \varepsilon\right\} \quad(p=1,2),
$$

then $\varepsilon_{3} \equiv \varepsilon_{1} \cdot \varepsilon_{2}$ is a set of positive measure.

Relation (3.9) may then be written in each of the forms

$$
\begin{array}{r}
R_{1}\left(y_{p}-h_{p}\right) \\
+\sum_{s=2}^{k} R_{s}\left(y_{p}-h_{p}\right) \exp \left\{-t_{s, m(j)} h_{p}\right\} \exp \left\{t_{s, m(j)} y_{p}\right\} \rightarrow 0 \\
\left(y_{p} \text { in } \varepsilon_{p}, p=1,2\right) .
\end{array}
$$

If we consider only points in $\varepsilon_{s}$, then $y_{1}$ and $y_{2}$ may be identified:

$$
\begin{array}{r}
R_{1}\left(y-h_{p}\right) \\
+\sum_{s=2}^{k} R_{s}\left(y-h_{p}\right) \exp \left\{-t_{s, m(j)} h_{p}\right\} \exp \left\{t_{s, m(j)} y\right\} \rightarrow 0 \\
\left(y \text { in } \varepsilon_{3}, p=1,2\right) .
\end{array}
$$

On subtracting we have

$$
\begin{array}{r}
{\left[R_{1}\left(y-h_{1}\right)-R_{1}\left(y-h_{2}\right)\right]+\sum_{s=2}^{k}\left[R_{s}\left(y-h_{1}\right) \exp \left\{-t_{s, m(j)} h_{1}\right\}\right.} \\
\left.-R_{s}\left(y-h_{2}\right) \exp \left\{-t_{s, m(j)} h_{2}\right\}\right] \exp \left\{t_{s, m(j)} y\right\} \rightarrow 0 \\
\left(y \text { in } \varepsilon_{3}\right) .
\end{array}
$$

Since $R_{1}(x)$ is of actual degree $Q$, and $Q>0$, we see that

$$
H(y) \equiv\left[R_{1}\left(y-h_{1}\right)-R_{1}\left(y-h_{2}\right)\right]
$$

is a polynomial of degree exactly $Q-1$, and is therefore not identically zero. But $H(y)$ being of degree less than $Q$, this places (3.13) in the category of cases for which the theorem is true, since now fewer than $\Lambda$ polynomials are of degree $Q$. Hence all coefficients approach zero. This is however contrary to the condition that $H(y) \not \equiv 0$.

We have thus arrived at a contradiction, so the assumption that Theorem 3.1 is false is untenable.

4. Higher dimensions. We shall now show that the foregoing results extend to the general case of $p$ dimensions. Throughout this section the term measure refers to p-dimensional measure. Proofs for 
the general case usually follow those of the preceding sections, and are accordingly given briefly or not at all.

LEMma 4.1. Let $\left\{u_{s, n}\right\}, s=1, \cdots, p$, be real sequences such that for at least one value of $s,\left\{u_{s, n}\right\}$ does not have zero as its limit. The relation

$$
\lim _{n \rightarrow \infty} \exp \left\{\sum_{s=1}^{p} u_{s, n} x_{s}\right\}=1
$$

cannot hold on a set of points $(x) \equiv\left(x_{1}, \cdots, x_{p}\right)$ of positive measure.

Assume that the lemma is false, so there is a set $\mathcal{F}$ of positive measure for which (4.1) holds. Suppose $s=q$ is the value for which $u_{q, n}$ does not approach 0 . We may then assume that $\left\{u_{q, n}\right\}$ does not have zero as limit point. If a subsequence $\{n(j)\}$ of $\{n\}$ exists for which all the sequences $\left\{u_{s, n(j)}\right\}, s=1, \cdots, p$, are bounded, then there will be a further subsequence $\{m(j)\}$ for which the following limits exist:

$$
\lim _{j \rightarrow \infty} u_{s, m(j)}=l_{s} \quad(s=1, \cdots, p),
$$

with $l_{q} \neq 0$. Hence if $(x)$ is in $\mathcal{F}$, then $(x)$ must satisfy one of the equations

$$
\frac{1}{2 \pi} \sum_{s=1}^{p} l_{s} x_{s}=0, \pm 1, \pm 2, \cdots .
$$

For each choice of the right side, (4.2) is a hyperplane, and is of measure zero. The totality of planes (4.2) is likewise of zero measure, and so, therefore, is $\mathcal{F}$, which is contrary to assumption.

There remains to consider the case where for at least one value of $s$, say $s=1$, and a subsequence $\{n(j)\}$,

$$
\left|u_{1, n(j)}\right| \rightarrow \infty \text {. }
$$

The remainder of the argument now follows that of Lemma 2.1, with obvious $p$-dimensional modifications.

LEMma 4.2. Let $\left\{t_{s, r, n}\right\}, s=1, \cdots, k ; r=1, \cdots, p$ be real sequences with the following property: $a$ value $r=q$ exists such that none of the sequences $\left\{t_{s, q, n}\right\},\left\{t_{s, q, n}-t_{\sigma, q, n}\right\}(s \neq \sigma)$ has zero as a limit point. If real or complex constants $\left\{A_{s, n}\right\}$ exist such that

$$
\sum_{s=1}^{k} A_{s, n}\left[\exp \left\{\sum_{r=1}^{p} t_{s, r, n} x_{r}\right\}-1\right] \rightarrow 0
$$

for all $(x) \equiv\left(x_{1}, \cdots, x_{p}\right)$ on a set $\varepsilon$ of positive measure, then 


$$
A_{s, n} \rightarrow 0
$$$$
(s=1, \cdots, k) .
$$

The proof is like that of Lemma 2.2 with simple modifications that need not be detailed here.

Lemma 4.2 leads directly to the following theorem.

TheOREM 4.1. Let $\left\{a_{s, n}\right\}, s=1, \cdots, k$, be real or complex sequences, and let the real sequences $\left\{q_{s, r, n}\right\}, s=1, \cdots, k ; r=1, \cdots, p$, be such that for some value $r=\omega$, none of the sequences $\left\{q_{s, \omega, n}-q_{\sigma, \omega, n}\right\}(s \neq \sigma)$ has zero as a limit point. If

$$
\sum_{s=1}^{k} a_{s, n} \exp \left\{\sum_{r=1}^{p} q_{s, r, n} x_{r}\right\} \rightarrow 0
$$

for all $(x) \equiv\left(x_{1}, \cdots, x_{p}\right)$ on a set $\mathcal{E}$ of positive measure, then

$$
a_{s, n} \rightarrow 0 \quad(s=1, \cdots, k) .
$$

The proof follows an earlier one (Theorem 2.1), as does the next result:

Corollary 4.1. Let the sequences $\left\{t_{s, r, n}\right\}$ satisfy the hypothesis of Lemma 4.2. If a constant $A$ and constants $\left\{a_{s, n}\right\}$ exist such that

$$
\sum_{s=1}^{k} a_{s, n} \exp \left\{\sum_{r=1}^{p} t_{s, r, n} x_{r}\right\} \rightarrow A
$$

for all ( $x$ ) on a set of positive measure, then

$$
A=0 ; \quad a_{s, n} \rightarrow 0 \quad(s=1, \cdots, k) .
$$

Finally, we have

Theorem 4.2. Let $\left\{q_{s, r, n}\right\}, s=1, \cdots, k ; r=1, \cdots, p$, be real sequences satisfying the hypothesis of Theorem 4.1. Let $\left\{P_{s, n}\left(x_{1}, \cdots, x_{p}\right)\right\}$ be real or complex polynomial sequences:

$$
\begin{array}{r}
P_{s, n}\left(x_{1}, \cdots, x_{p}\right)=\sum_{h_{1}+\cdots+h_{p}=0}^{e_{s}} a_{s, n ; h_{1}, \cdots, h_{p}} x_{1}^{h_{1}} \cdots x_{p}^{h_{p}} \\
(s=1, \cdots, k),
\end{array}
$$

in which $e_{s}$ is independent of $n$. If

$$
\sum_{s=1}^{k} P_{s, n}\left(x_{1}, \cdots, x_{p}\right) \exp \left\{\sum_{r=1}^{p} q_{s, r, n} x_{r}\right\} \rightarrow 0
$$

for all ( $x$ ) on a set $\mathcal{E}$ of positive measure, then 


$$
a_{s, n ; h_{1}, \cdots, h_{p}} \rightarrow 0 \quad\left(0 \leqq h_{1}+\cdots+h_{p} \leqq e_{s} ; s=1, \cdots, k\right) .
$$

$\mathrm{Up}$ to a point the proof is patterned after that of Theorem 3.1. When the equivalent of (3.13) is obtained, however, we can no longer assert that $H\left(y_{1}, \cdots, y_{p}\right)$ is not identically zero simply from the fact that two distinct sets $(h)_{1}=\left(h_{11}, \cdots, h_{1 p}\right),(h)_{2}=\left(h_{21}, \cdots, h_{2 p}\right)$ exist such that

$$
\begin{aligned}
H\left(y_{1}, \cdots, y_{p}\right) \equiv & R_{1}\left(y_{1}-h_{11}, \cdots, y_{p}-h_{1 p}\right) \\
& -R_{1}\left(y_{1}-h_{21}, \cdots, y_{p}-h_{2 p}\right) .
\end{aligned}
$$

In fact, nonconstant polynomials in more than one variable exist that are "periodic." We avoid this difficulty by observing that for a fixed point $(h)_{1}$, the point $(h)_{2}$ can be chosen arbitrarily on a set of positive measure. Examination of the proof of Lemma 2.2 shows this. Now if a polynomial $L\left(x_{1}, \cdots, x_{p}\right)$ has the property that

$$
L\left(x_{1}+c_{1}, \cdots, x_{p}+c_{p}\right) \equiv L\left(x_{1}, \cdots, x_{p}\right)
$$

for all sets $(c) \equiv\left(c_{1}, \cdots, c_{p}\right)$ on a set of positive measure, then surely $L \equiv$ constant.

In our case, therefore, if $H\left(y_{1}, \cdots, y_{p}\right) \equiv 0$ for all possible choices of $(h)_{2}$, then $R_{1}$ is a constant, contrary to the fact that its degree is $Q>0$ (cf. Theorem 3.1). The remainder of the proof offers no difficulty.

The Pennsylvania State College 